\title{
Multilayer flows in molecular networks identify biological modules in the human proteome
}

\author{
Giuseppe Mangioni ${ }^{1, \dagger}$, Giuseppe Jurman ${ }^{2, \star}$, and Manlio De Domenico ${ }^{2, *}$, \\ ${ }^{1}$ Universitá degli Studi di Catania, V.le A. Doria 6, 95125, Catania, Italy \\ ${ }^{2}$ Fondazione Bruno Kessler, Via Sommarive 18, 38123 Povo (TN), Italy
}

\begin{abstract}
A variety of complex systems exhibit different types of relationships simultaneously that can be modeled by multiplex networks. A typical problem is to determine the community structure of such systems that, in general, depend on one or more parameters to be tuned. In this study we propose one measure, grounded on information theory, to find the optimal value of the relax rate characterizing Multiplex Infomap, the generalization of the Infomap algorithm to the realm of multilayer networks. We evaluate our methodology on synthetic networks, to show that the most representative community structure can be reliably identified when the most appropriate relax rate is used.

Capitalizing on these results, we use this measure to identify the most reliable meso-scale functional organization in the human protein-protein interaction multiplex network and compare the observed clusters against a collection of independently annotated gene sets from the Molecular Signatures Database (MSigDB). Our analysis reveals that modules obtained with the optimal value of the relax rate are biologically significant and, remarkably, with higher functional content than the ones obtained from the aggregate representation of the human proteome. Our framework allows us to characterize the meso-scale structure of those multilayer systems whose layers are not explicitly interconnected each other - as in the case of edge-colored models - the ones describing most biological networks, from proteomes to connectomes.
\end{abstract}

\section{INTRODUCTION}

A variety of natural and artificial systems can be described in terms of interactions and relationships among their constituents, which find a suitable representation in terms of complex networks [1].

Despite their wide applicability, single-layer complex networks are not able to capture the complexity of many empirical systems, as the ones where units exhibit multiple types of relationships simultaneously. This is the case of social systems, where an individual can have family, business or trust interactions with other individuals, or of transportation systems, where geographical areas might be connected by different transporation means such as bus, tube, rail, so forth and so on.

The suitability of multilayer networks for capturing this higher amount of complexity led to a growing interest in their study [2], [3], [4] and to a more general mathematical framework, which can be used when nodes are connected to each other via multiple types of edges or a network changes in time [5]. In fact, multilayer networks are more adequate to model real world interactions that cannot be aggregated into a single network without a loss, in general, of some important structural or dynamical properties [6], [7] (see Fig. 1 for an illustration of the type of networks considered in this study).

One of the most attractive problems in network science deals with the identification of the so-called meso-scale structure of a complex network, a topic of intensive research activity across multiple disciplines [8]. Its importance relies in the ability to unveiling communities of units that, in turn, can be used to explain some hidden behaviours of networks

† giuseppe.mangioni@dieei.unict.it, * jurman@fbk.eu,*mdedomenico@fbk.eu emerging as the result of the complex interaction patterns among nodes (or entities).

Community detection has been successfully used to analyze the structure of single-layer networks and for modeling several kinds of interactions, such as social relationships, genetic interactions among biological molecules or trade among countries [9], [10], [11], [12], [13], [14], [15], just to mention a few (a detailed introduction to communities in networks can be found in [16], [17]).

In the case of biological systems, community analysis has been used to identify structural and functional modules, in order to determine molecules with similar biological function within a cell and to improve our understanding of life and disease [18], [19], [20], [21]. Proteins are molecular building blocks of a cell which play special roles (e.g., catalysis, signalling, etc.) for its modular function and hierarchical organization. In fact, mutation of single genes or altered activation/inhibition regulation quickly propagate to perturbate the protein-protein interaction (PPI) network, causing abnormal functions in tissues and organs that might culminate in diseases. Multilayer network modeling is expected to provide a framework more suitable than traditional aggregated approaches for the analysis of molecular systems and, more specifically of PPI networks. In fact, multilayer networks allow for the integration of multiple information sources, without neglecting or heuristically aggregating different types of interactions among biological units of possibly different type [22], providing a more realistic framework for systems biology.

Aggregate (also known as "monoplex") PPI networks have been successfully used to correlate the cellular function of single proteins with their topological role [23], reveal- 
ing indispensable proteins, from a network controllability perspective, that turned out to be commonly targeted by disease-causing mutations and human viruses or have been identified as drug targets |24|. Evidence for a strict relationship between their mesoscale organization and functional segregation within a cell has been provided for both human and non-human organisms [25], [26], [27], thus increasing our understanding of functional relationships with genetic disorders [28] and cancer [29].

In this work, we explore the suitability of multilayer community detection for the analysis of PPI interactions, with special focus on the human proteome. The paper is organized as in the following. First, we present the community detection problem in network science and briefly introduce Multiplex Infomap, the methodology used in this work. Second, we introduce the information-theoretic measure - namely the normalized information loss - used in this study to select the relax rate, i.e., the parameter characterizing Multiplex Infomap in the analysis of noninterconnected multiplex networks. Third, we present the analysis of synthetic networks to validate the goodness of this measure in determining a suitable relax rate. Finally, we apply the proposed methodology to unravel the mesoscale functional organization of the human PPI multiplex network and we validate the discovered modules against a collection of annotated gene sets.

\section{Community detection in multilayer net- WORKS}

Informally, a community is a group of nodes more densely connected each other inside the group and sparsely connected to nodes outside the group. Despite this intuitive concept, a precise definition of a community is still a topic of debate among network scientists. One of the most adopted formulation is based on the definition of the so-called nullmodel, i.e. a model to which the network can be statistically compared to random expectation in order to assert the existence of any degree of modularity. Starting from the definition of a specific null-model - i.e., a random network satisfying certain requirements, such as preserving the number of nodes, the number of links and the degree distribution of the original network - a modularity function to measure the quality of a given partition has been introduced in Ref. [30], [31]. Despite some limitations [32], |33], the modularity function has been successfully used as a quality measure to evaluate a given network partition and as a cost function to be optimized to uncover communities [34], [35], [36]. Furthermore, the original modularity definition has been extended to directed [37], weighted [38], bipartite [39] networks and to evaluate partitions with overlapping communities [40].

Other than modularity based methods, in literature there exists a lot of alternative approaches to solve the problem of community discovering [16], [17]. For example, in Ref. [41] an information-theoretic based method is presented. This method is based on the formulation of a new quality function called map equation [42], which allows to find the optimal description of the network by compressing its information flow. The algorithm is the core of Infoman ${ }^{1}$

1. http://www.mapequation.org/

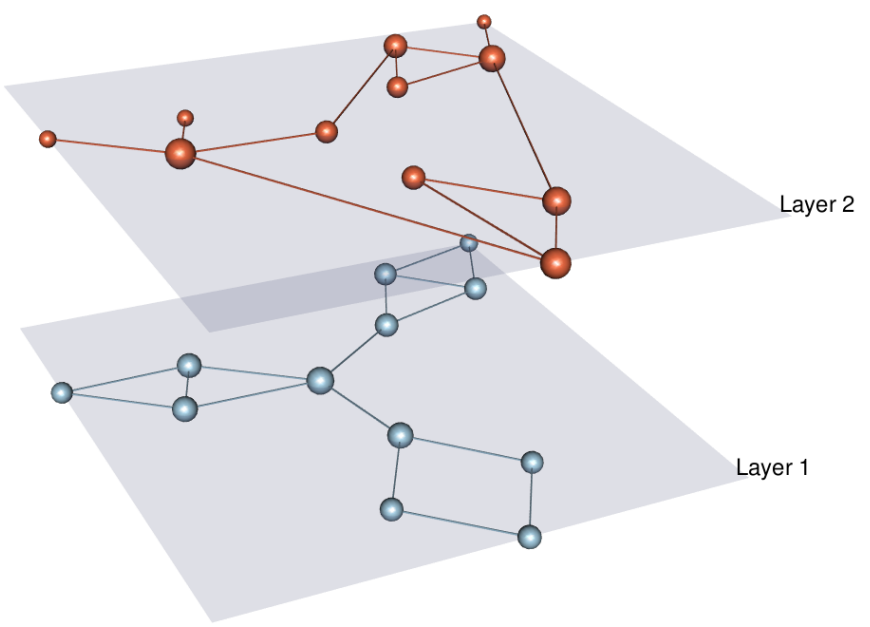

Fig. 1: Illustration of a non-interconnected multiplex network (with 2 layers), also called edge-colored multigraph, a special type of multilayer network. This model is defined by i) the existence of a non-empty set of nodes which is common to multiple layers and ii) the absence of explicit information about inter-layer links between node's replicas across layers. The second feature often requires to use a free parameter to modulate the coupling between layers for analytical purposes.

the search method for minimizing the map equation over possible network partitions.

Many methods and measures developed for single layer networks have been extended to be applicable to multilayer networks [5], [43], [44], [45], [46]. In this context new community detection methods have been devised, mainly by reusing concepts already developed for single layer networks. In Ref. [47], the authors proposed a method based on a generalization of the modularity to multilayer networks. This extended modularity is mainly based on generalized null models obtained by considering a Laplacian dynamics |48], |49| on the multilayer network. To compute communities by using such a generalization of the modularity function, an extension of the Louvain algorithm [50] has been also proposed in Ref. [51].

In Ref. [52 an extension of the map equation to multilayer networks is introduced. It is based on the generalization of random walks to multilayer systems [53], which in turn are used to generate the corresponding network flow to be compressed in order to identify community flows in multilayer networks. The resulting algorithm - i.e. Multiplex Infomap [52] - is the extension of Infomap to the case of multilayer networks.

A drawback of community detection algorithms for noninterconnected/edge-colored networks - i.e., systems where inter-layer connectivity is not defined - is their dependence on at least one parameter which regulates the structural or dynamical coupling between layers. In the case of Multiplex Infomap, this parameter is known as the relax rate $r$. The relax rate is the parameter responsible of modeling movement among layers. At each step of the underlying random walk, there is a $1-r$ probability that the random walker simply moves to a neighbour in the same layer, and an $r$ probability 
that it changes layer, and then moves to a neighbour on that layer.

To better understand this concept, let us denote by $W_{i j}^{\beta}$ the intra-layer adjacency matrix representing the connectivity of nodes within a generic layer $\beta$ of the multiplex system. Let $D_{i}^{\alpha \beta}$ represent the inter-layer adjacency matrix of a physical node $i$, encoding the connectivity of that node with its replicas on other layers. Let $S_{i}^{\alpha}=\sum_{\beta} D_{i}^{\alpha \beta}$ indicate the inter-layer out-strengths and let $s_{i}^{\beta}=\sum_{j} W_{i j}^{\beta}$ indicate the intra-layer out-strengths of node $i$ in layer $\alpha$ and $\beta$. It follows that the probability of a random walker to move, in general, from node $i$ in layer $\alpha$ to node $j$ in layer $\beta$, is given by $[52$

$$
\mathcal{P}_{i j}^{\alpha \beta}(r)=(1-r) \delta_{\alpha \beta} \frac{W_{i j}^{\beta}}{s_{i}^{\beta}}+r \frac{W_{i j}^{\beta}}{\sum_{\beta} s_{i}^{\beta}} .
$$

The choice of $r$ is crucial and, in general, it depends on the network under analysis. While empirical results suggest that values smaller than 0.5 are generally appropriate for most networks [52], finding the actual optimal value is still an unsolved problem. Moreover, in community detection the concept of an absolute optimal simply does not exist, as it is difficult to ascertain whether the chosen algorithm is able to detect the absolute optimal partition. In fact, a safer approach is to assess that a certain partition can be optimal with respect to a specific algorithm. In this study, our goal is to find the value of $r$ which provides the best possible partition with respect to Multiplex InfoMap in the case of multilayer systems where the strength of coupling among layers is unknown. As thoroughly explained in [54], the problem of finding the best possible partition in community detection is ill-defined - in the case of multiplex networks and, more generally, in complex networks - and the result of the proposed procedure will provide optimal partitions with respect to the information-theoretic quality functions used in this work.

\section{INFORMATION-THEORETIC APPROACH TO PA- RAMETER SELECTION}

Multiplex InfoMap is an algorithm which optimizes the map equation [41], a measure of the information-theoretic duality between data compression and the problem of extracting significant information from compressed data. Given that its roots lie firmly in the realm of information theory, it is natural to develop an information-theoretic algorithm to determine the relax rate producing an optimal partition, with respect to some criteria. In the domain of information theory, this partition would be the one which retains the most information about the network inside the communities. In literature, there are several attempts at exploiting the concepts of information theory to evaluate the quality of a partition [41], [55], [56], [57]. Here, we investigate the suitability of information loss [56 for optimal selection of relax rate.

Information loss occurs when a certain source of information is compressed in a way that some of the information is discarded as a result of the compression. Since any source of information can be fed to compression algorithms, we are now going to describe how to compress a network, and the information loss that derives from this operation.

Compressing a network $X$ involves finding some representation $Y$ that only keeps part of the available information on connectivity. In the following, we indicate by $H(X)$ the information entropy of the random variable $X$ encoding the original network connectivity, while we indicate by $H(Y)$ the information entropy of the random variable $Y$, which provides a simplified and coarse-grained representation of the system. As we will see in the following, an explicit calculation of $H(X)$ and $H(Y)$ is not required. In [56], the authors compress the network into a representation that preserves the information contained inside the communities in order to evaluate how much information is required to rebuild $X$ given the representation $Y$. If we name this quantity $H(X \mid Y)$, given that $H(X)$ is the average amount of information required to describe $X$, we can compute it from mutual information $I(X ; Y)$ between $X$ and $Y$ by

$$
H(X \mid Y)=H(X)-I(X ; Y) .
$$

The compressed representation $Y$ is still a graph where each node is a community and links between nodes are inter-community connections. Hence, we may completely describe $Y$ with the tuple $(N, L)$, with $N=\left\{n_{i}\right\}$, where $n_{i}$ is the number of nodes of the $i$-th community, and $L=\left\{l_{i j}\right\}$, where $l_{i j}$ is the number of links that go from community $i$ to community $j$. Note that the definition provided is exactly equivalent to the cross-entropy (or negative log-likelihood) of the stochastic block model (SBM) [58], a widely adopted generative model for random graphs. If we assume that there are $m$ communities, in the simplest case of undirected and unweighted networks, Eq. (2) reduces to:

$$
H(X \mid Y)=\log _{2}\left[\prod_{i=1}^{m}\left(\begin{array}{c}
n_{i}\left(n_{i}-1\right) / 2 \\
l_{i i}
\end{array}\right) \prod_{j<i}\left(\begin{array}{c}
n_{i} n_{j} \\
l_{i j}
\end{array}\right)\right] .
$$

This formula accounts for all the possible ways to arrange the links that go from nodes of community $i$ to nodes of community $j$, hence representing all the possible configurations of networks that can be reconstructed knowing $Y$. The higher the value, the more information is contained in the inter-community links. Extending this formula for directed networks is straightforward, since one should evaluate the possibility that a link can connect two nodes in two different ways (from $i$ to $j$ and vice-versa):

$$
H(X \mid Y)=\log _{2}\left[\prod_{i=1}^{m}\left(\begin{array}{c}
n_{i}\left(n_{i}-1\right) \\
l_{i i}
\end{array}\right) \prod_{j \neq i}\left(\begin{array}{c}
n_{i} n_{j} \\
l_{i j}
\end{array}\right)\right] .
$$

Weights can be included as well, to account for more complex structures. For each link $l_{i j}$ of $Y$, which represents the total number of links from community $i$ to community $j$, we have a quantity $w_{i j}$ encoding the sum of the weights of links that go from $i$ to $j$. Ideally, each configuration reconstructed from $Y$ using Eq. (4) generates further configurations if we consider all the possible ways to distribute $w_{i j}$ among $l_{i j}$ links. The number of those configurations is infinite if the weights are real numbers: given a weight $w_{i j}$, the problem is analogous to splitting the interval $\left[0, w_{i j}\right]$ in $l_{i j}$ parts, and since any real interval is uncountable, 


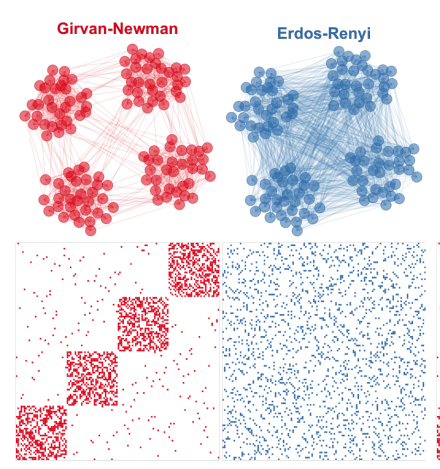

(a) GN/ER

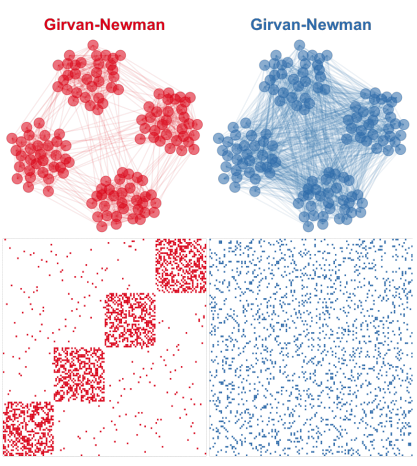

(b) GN/GN $10 \%$

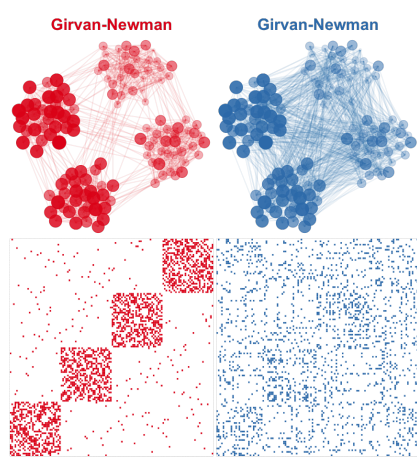

(c) GN/GN $50 \%$

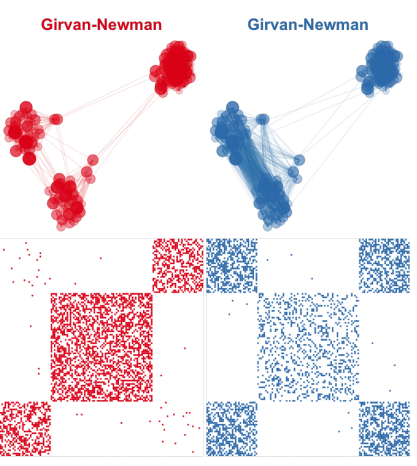

(d) GN/cGN

Fig. 2: Synthetic multiplex network models considered in this study as benchmarks. Network of 128 nodes and different community structures across layers are shown. (a) A layer with a strong community structure (Girvan-Newman model) coupled to a layer with a homogeneous structure (Erdos-Renyi model) playing the role of structural noise. (b) Two GirvanNewman networks with $10 \%$ of nodes belonging to overlapping communities across layers. (c) As in (b), but with $50 \%$ overlapping community structure. (d) A layer with a network generated by using the Girvan-Newman model (GN) coupled to a layer with the complementary network (cGN). See the main text for further details.

there are infinite ways to make the partition. However, if we impose the restriction that the weights are natural numbers, the number of partitions can be calculated as follows. First, we assign the weight 1 to each one of the $l_{i j}$ links, thus imposing the restriction $w_{i j} \geq l_{i j}$. Since we already distributed $l_{i j}$ out of the total $w_{i j}$, calculating all possible distributions of the remaining $w_{i k}-l_{i j}$ among $l_{i j}$ links depends on combinations with replacement:

$$
\begin{aligned}
C^{R}\left(l_{i j}, w_{i j}-l_{i j}\right) & =\frac{\left(l_{i j}+w_{i j}-l_{i j}-1\right) !}{\left(w_{i j}-l_{i j}\right) !\left(l_{i j}-1\right) !} \\
& =\frac{\left(w_{i j}-1\right) !}{\left(w_{i j}-l_{i j}\right) !\left(l_{i j}-1\right) !} \\
& =\left(\begin{array}{c}
w_{i j}-1 \\
l_{i j}-1
\end{array}\right) .
\end{aligned}
$$

Thus, we can update equation Eq. (4) to include all the possible ways to distribute $w_{i j}$ among $l_{i j}$ links:

$$
\begin{aligned}
H(X \mid Y)= & \log _{2}\left[\prod_{i=1}^{m}\left(\begin{array}{c}
n_{i}\left(n_{i}-1\right) \\
l_{i i}
\end{array}\right)\left(\begin{array}{c}
w_{i i}-1 \\
l_{i i}-1
\end{array}\right) \times\right. \\
& \left.\times \prod_{i \neq j}\left(\begin{array}{c}
n_{i} n_{j} \\
l_{i j}
\end{array}\right)\left(\begin{array}{c}
w_{i j}-1 \\
l_{i j}-1
\end{array}\right)\right] .
\end{aligned}
$$

A more general formula, accounting for the possibility of self-links (e.g., useful for modeling citation networks) is given by

$$
H(X \mid Y)=\log _{2}\left[\prod_{i=1}^{m} \prod_{j=1}^{m}\left(\begin{array}{c}
n_{i} n_{j} \\
l_{i j}
\end{array}\right)\left(\begin{array}{c}
w_{i j}-1 \\
l_{i j}-1
\end{array}\right)\right] .
$$

Since $H(X \mid Y)$ represents the information that is lost when compressing the network, a good compression requires $H(X \mid Y)$ to be as small as possible: hence, our goal is to minimize this quantity. It is worth remarking that it is possible to learn the latent block structure in presence of realvalued weights through the use of a parametric distribution, as shown in [59], by exploiting the fact that the proposed measure can be interpreted as a log-likelihood of a SBM.
For practical applications, it might be useful to define a standardized version of this measure. If we name the information loss $H_{r}(X \mid Y)$ for a certain relax rate $r$, we define its normalized version as:

$$
H_{r}^{*}(X \mid Y)=\frac{H_{r}(X \mid Y)-\min _{0<r \leq 1} H_{r}(X \mid Y)}{\max _{0<r \leq 1} H_{r}(X \mid Y)-\min _{0<r \leq 1} H_{r}(X \mid Y)} .
$$

\section{ANALYSIS OF SYNTHETIC NETWORK MODELS}

To better understand the suitability and the limitations of the the proposed measure, we analyze a set of synthetic benchmark networks. The multiplex toy models consist of nodes which are connected in different ways on two layers, while inter-layer connectivity is not given explicitly. We consider four kinds of benchmarks:

- GN/ER. This system is generated by combining an Erdos-Renyi layer with one generated by the GirvanNewman (GN) benchmark [9]. A community structure is only present in the GN layer, hence this multiplex network is used to test the impact of coupling noise to a structured population.

- GN/GN. This system consists of two GN networks, with tunable cross-layer community overlap, generated as following. First, we create a single-layered GN network and duplicate it to generate a multiplex network with two layers. Then, in second layer, we iteratively swap the neighbours of pairs of randomly selected nodes to change the community structure. The swapping procedure is repeated until the ratio of community overlapping across layers, defined by the fraction of nodes that belong to the same communities across layers, is reached. Two classes of networks are generated, corresponding to a different amount of overlapping across layers (50\% and 10\%, respectively).

- GN/CGN. This system consists of two layers, one GN network and one complementary GN (CGN). In order to explain how these two layers are generated, 
Number of communities

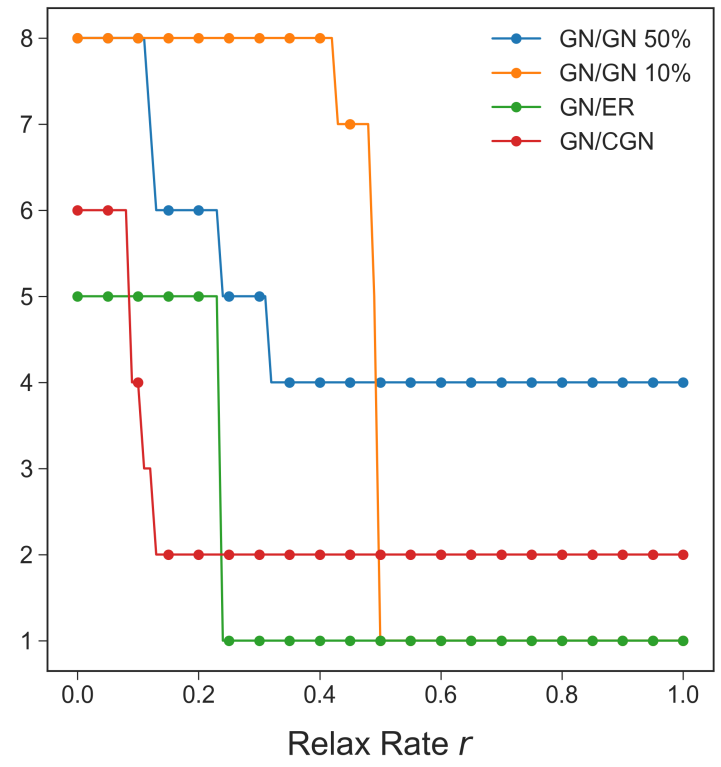

(a)
Normalized information loss

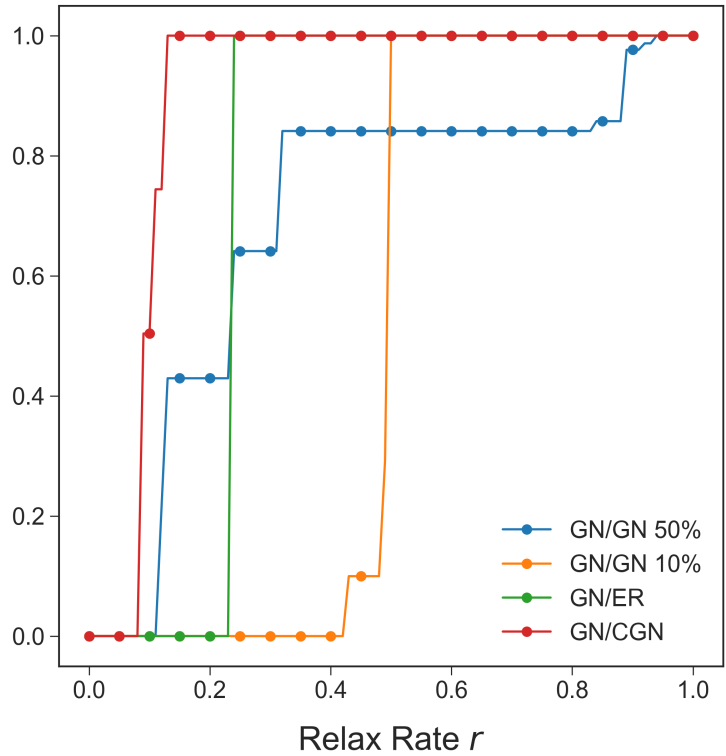

(b)

Fig. 3: (a) Number of communities and (b) normalized information loss, for different synthetic network models, while varying the value of the relax rate.

let's suppose that communities in each layer are labeled with the integers $\{1,2,3,4\}$. In the first layer, we preserve the structure of communities 1 and 2, while nodes in communities 3 and 4 are randomly rewired with a given probability $p=0.5$. A fraction of $10 \%$ of nodes in communities 3 and 4 are then connected to nodes in communities 1 and 2 chosen at random. The same algorithm is then applied to layer 2 , but preserving communities 3 and 4 and randomly rewiring nodes in communities 1 and 2 .

These synthetic network models (see Fig. 2) are used to test the ability of our methodology to detect the most relevant community structure among the ones identified by Multiplex Infomap for varying relax rates. Results are shown in Fig. 3 Our analysis highlights some special properties of normalized information loss when an underlying community structure is present or absent. First, it is worth remarking that transitions between two or more regimes are always observed: in fact, the relax rate acts as a dynamical multiresolution parameter, allowing to identify communities at different dynamical scales. Let us consider, for instance, the GN/ER model in Fig. 2a: for $r$ smaller than 0.25 we identify 5 communities (the four planted partitions in the GN layer plus the ER layer, acting as a single community), whereas a sharp transition towards 1 community is observed above that value. In this latter case, the random walkers are exploring the ER layer too often and the relevance of the strong community structure planted in the GN is washed out. While this behavior finds a clear explanation, it might be useful for applications to identify a dynamical scale, by means of a suitable value of the relax rate, where the identified meso-scale organization is more representative of the system. Here, the normalized information loss we have previously introduced plays a crucial role: in fact, it is zero before the transition point and explosively increases to one above it (see Fig. 2p). Therefore, our measure is highlighting that, from an information-theoretic perspective, the meso-scale found below the transition point provides more information about the system.

Similar arguments can be given to explain the behavior of transitions in the other network models considered in the same figure. The transition point can change, depending on the model and its complexity, and we might observe more than a single sharp transition point, each one identifying a transition between different dynamical regimes. Remarkably, in all cases the evolution of normalized information loss resembles the evolution of the number of communities while providing, at the same time, a quantitative measure which helps to identify the range of dynamics describing the system with minimum information loss.

\section{Analysis of the Homo Sapiens PPI Multi- PLEX NetWorK}

In agreement with previous studies [6], [60], we analyze multiplex molecular networks built from the BioGRID (Biological General Repository for Interaction Datasets [61]) a public database with data compiled through comprehensive curation efforts, that stores and disseminates genetic and protein interaction information about simple organisms and human ${ }^{2}$ ). The curated set used in this study (BioGRID 3.4.155, updated to December 2017) consists of physical and genetic relations including interactions, chemical associations, and post-translational modifications (PTM) from 63,959 publications, leading to $1,168,521$ non-redundant interactions, 1,507,991 raw interactions, 11,820 non-redundant

\footnotetext{
2. http://thebiogrid.org
} 


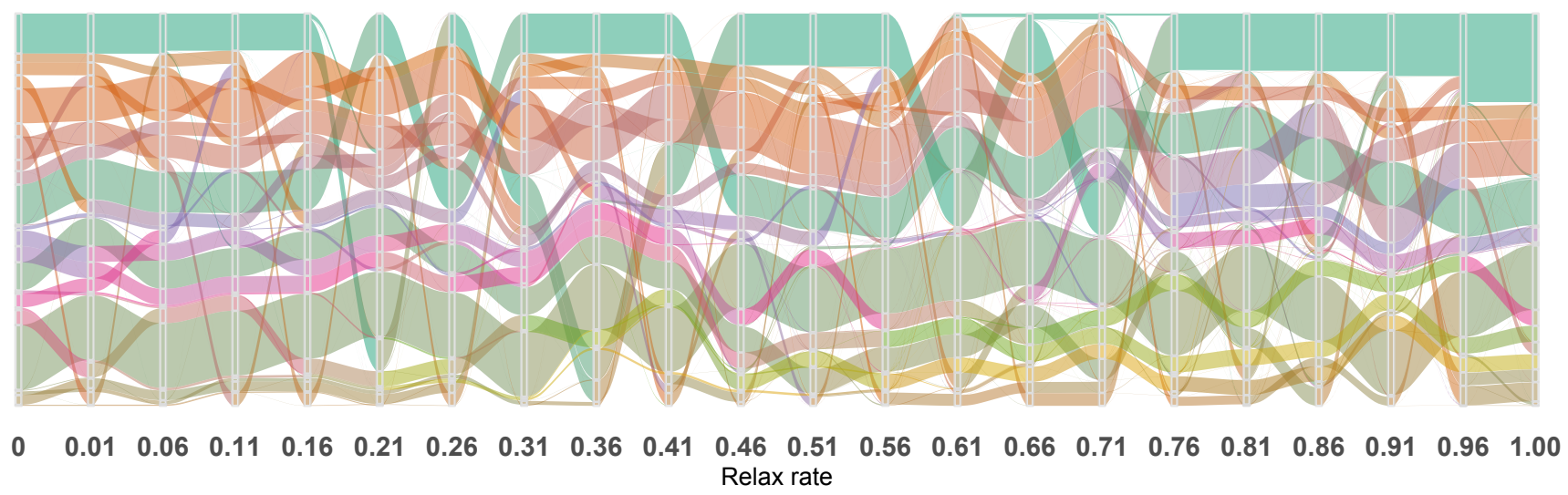

Fig. 4: Changes in the meso-scale organization of human multiplex proteome for varying relax rate. Clusters with at least 100 proteins are considered for clarity. This alluvial plot shows how partitions split and merge for increasing rate: larger clusters are quite stable, highlighting that differences in meso-scale are mostly due to smaller sets of proteins.

chemical associations, 27,785 raw chemical associations, 19,981 Unique PTM Sites, and 18,578 Un-Assigned PTMs.

More specifically, we focussed our attention on homo sapiens. In this data set, the networks represent PPI of two types, namely genetic and physical, and the layers correspond to a finer classification into seven types of interaction of different nature, i.e., physical, direct, co-localization, association, and suppressive, additive or synthetic genetic interaction. The total number of proteins in the network is 21,591 , linked by 338,113 interactions across all layers.

To compare our results against more traditional approaches based on the study of the aggregate representation of the same system, we build the corresponding aggregate network which consists of 315,766 unique weighted interactions. It is worth noting that $6.6 \%$ of the total number of interactions are present in at least two different layers.

First, we identify the meso-scale organization of the multiplex representation of the human proteome for varying relax rate. In Fig. 4 , we show how the identified communities split and merge for different values of $r$ : larger clusters are quite stable, highlighting that differences in meso-scale are mostly due to smaller sets of proteins. Figure 5 shows how the distribution of clusters' size changes depending on relax rate, providing a different analysis of the human proteome meso-scale organization. The number of very small clusters (size smaller than 2) decreases whereas larger functional clusters tend to form for increasing relax rate.

Both the multiplex and the aggregate representations are then tested for the biological functional content of their functional clusters through a standard enrichmentbased strategy using the Molecular Signatures Databas $e^{3}$ (MSigDB) [62], [63], |64]. In the current version 6.1 (Oct 2017), MSgiDB includes 17,786 gene sets divided into 8 major collections to be used as the knowledge base for enrichement studies. To obtain a quantitative assessement of the functional content associated to the sets of clusters identified from the multiplex network - for varying relax rate - or its aggregate representation, we adopt the follow-

3. http://software.broadinstitute.org/gsea/msigdb/collections.jsp ing procedure: given a set of clusters $\mathcal{C}$ and the $\operatorname{MSigDB} \mathcal{M}$, we compute the total functional content as the cumulative fraction of genes shared between the clusters and the collections of annotated gene sets:

$$
\operatorname{TFC}(\mathcal{C})=\sum_{\substack{C \in \mathcal{C},|C| \geq 10 \\ M \in \mathcal{M}}} J(C, M)=\sum_{\substack{C \in \mathcal{C},|C| \geq 10 \\ M \in \mathcal{M}}} \frac{C \cap M}{C \cup M},
$$

where $J(\cdot, \cdot)$ is the Jaccard index [65], |66]. It is worth noting that in our analysis we have considered only clusters with at least 10 genes to allow for statistically meaningful enrichment analysis.

The plot of the total functional content as a function of the relax rate is shown in the top panel of Fig. 6 the functional content of the set of clusters is decreasing for increasing value of the relax rate, and for relax rates close to one, the content coincide with the functional content of the clusters obtained in the aggregate case. Overall then the largest biological meaningfulness is reached in the multiplex case, for relax rates close to zero, i.e., when random walkers exploring the multiplex systems do not switch layer frequently. This results is remarkable because in perfect agreement with the range of $r$ where normalized information loss is minimum (middle panel of Fig. 6.

\section{Conclusions}

In this paper, we have proposed an information-theoretic approach for parameter selection in community detection analysis performed with the Multiplex Infomap algorithm, the multilayer variant of the well-known Infomap algorithm. In fact, in the specific case of non-interconnected (i.e., edge-colored) networks, Multiplex Infomap depends on the relax rate, which is responsible for coupling the layers of the network and allows to study the system at multiple dynamical scales.

To tackle the problem of selecting an optimal value for this parameter, our work starts from the observation that the information content of a complex network can be fully encoded by its adjacency matrix representation. In a complex 


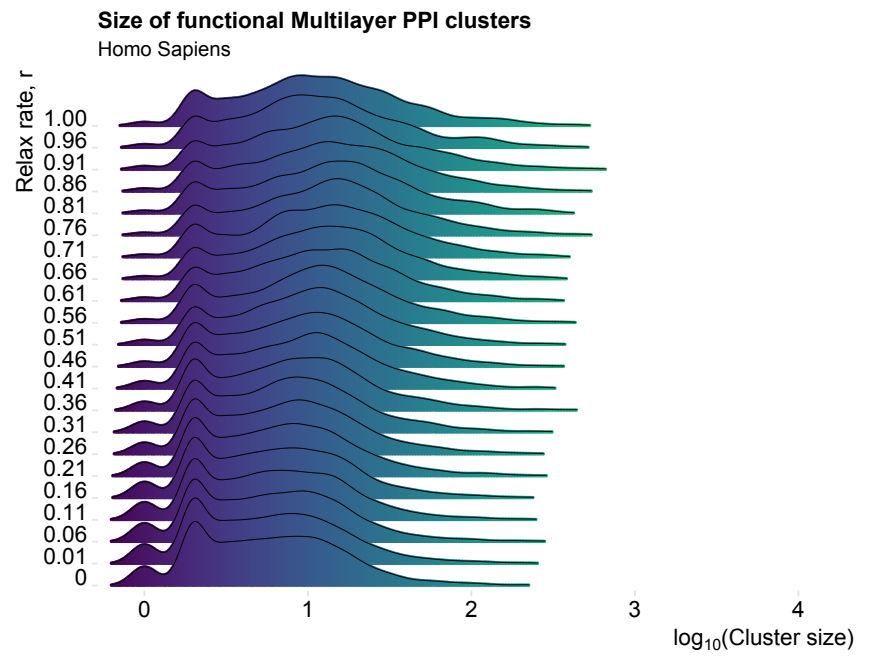

Fig. 5: Changes in the meso-scale organization of human multiplex proteome: the distribution of clusters' size is shown for varying relax rate. For increasing relax rate, the number of very small clusters (size smaller than 2) decreases while larger functional clusters of proteins tend to form.

network with a community structure, we can distinguish between information retained within communities, encoded by intra-community links, and information retained among different communities, encoded by inter-community links. Since a good partition of the system is expected to keep within the same community most of the links, or the information flow, we have shown that it is possible to find the most reliable partitions by minimizing the information contained in inter-community connectivity. We achieved this goal by evaluating one information-theoretic measure, namely the normalized information loss.

We have analyzed the behavior of this measure for varying relax rate in both synthetic and empirical networks. Results from trivial benchmarks confirmed our expectations, whereas results from toy models with a non-trivial multiplex community structure show that optimal values of the relax rate are range between 0.1 and 0.5 , the region of the parameter space where inter-layer coupling - indirectly caused by random walkers switching among layers - is strong enough for multilayer effects to become significant.

We have applied the proposed framework to the multiplex human proteome, consisting of proteins interacting physically and genetically. We have identified seven different layers by using the Biological General Repository for Interaction Datasets (BioGRID), in agreement with previous studies. Our analysis of this multiplex PPI network and of its aggregate representation has highlighted the existence of a range of relax rates close to zero where the information content of the meso-scale organization is maximum or, equivalently, where the information loss is minimum. To validate the identified functional clusters, we have performed an independent analysis by performing a biological enrichment through the well known Molecular Signatures Database (MSigDB), a huge collection of manually curated gene sets with known cellular function. Remarkably, the enrichment analysis provides a maximum functional content, which is

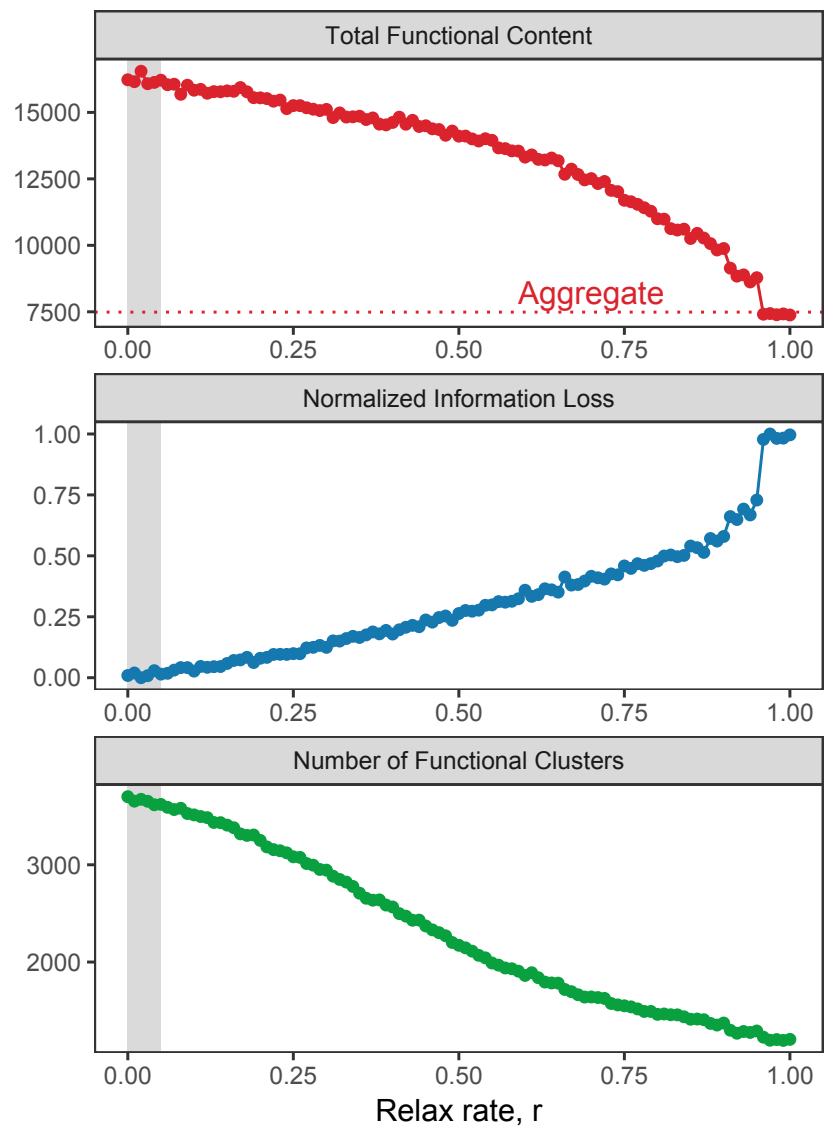

Fig. 6: Analysis of the meso-scale organization of genes in the human proteome. Top panel: total functional content of the cluster collections as a function of the relax rate, computed as the sum of the Jaccard indices of the intersections between each cluster and each gene set in MSigDB (see the text for details). The content is monotonically decreasing for increasing relax rate: for $r \approx 1$, the functional content coincides with the functional content of the cluster collection obtained from the aggregate representation of the system. Middle panel: normalized information loss (Eq. (8)) for increasing relax rate, highlighting an increasing degradation of the information content. Bottom panel: decreasing number of identified functional clusters for increasing relax rate. In all panels, the shaded area highlights the range of relax rates where normalized information loss is minimum which, remarkably, coincides with the range where the biological enrichment of identified clusters exhibits maximum functional content.

biologically significant, for the same range of relax rates where normalized information loss is minimum.

Our study provides a quantitative approach for the selection of a suitable value of relax rate in multilayer community detection on non-interconnected multiplex networks. The application of our framework to the study of human proteome provides results which outperform traditional approaches, such as the ones based on the analysis of aggregate representations of multiplex systems. Future applications of our framework include the analysis of different biological systems, from other organisms' multiplex 
PPI networks to the human brain.

\section{REFERENCES}

[1] S. Boccaletti, V. Latora, Y. Moreno, M. Chavez, and D.-U. Hwang, "Complex networks: Structure and dynamics," Physics reports, vol. 424, no. 4, pp. 175-308, 2006.

[2] M. Kivel, A. Arenas, M. Barthelemy, J. P. Gleeson, Y. Moreno, and M. A. Porter, "Multilayer networks," Journal of Complex Networks, vol. 2, no. 3, p. 203, 2014.

[3] S. Boccaletti, G. Bianconi, R. Criado, C. del Genio, J. GómezGardeñes, M. Romance, I. Sendiña-Nadal, Z. Wang, and M. Zanin, "The structure and dynamics of multilayer networks," Physics Reports, vol. 544, no. 1, pp. 1 - 122, 2014, the structure and dynamics of multilayer networks.

[4] M. De Domenico, C. Granell, M. A. Porter, and A. Arenas, "The physics of spreading processes in multilayer networks," Nature Physics, vol. 12, pp. 901-906, 2016.

[5] M. De Domenico, A. Solé-Ribalta, E. Cozzo, M. Kivelä, Y. Moreno, M. A. Porter, S. Gómez, and A. Arenas, "Mathematical formulation of multilayer networks," Physical Review X, vol. 3, no. 4, p. 041022, 2013.

[6] M. De Domenico, V. Nicosia, A. Arenas, and V. Latora, "Structural reducibility of multilayer networks." Nature communications, vol. 6, p. 6864, 2015.

[7] M. Diakonova, V. Nicosia, V. Latora, and M. San Miguel, "Irreducibility of multilayer network dynamics: the case of the voter model," New Journal of Physics, vol. 18, no. 2, p. 023010, 2016.

[8] M. E. Newman, "Communities, modules and large-scale structure in networks," Nature Physics, vol. 8, no. 1, pp. 25-31, 2012.

[9] M. Girvan and M. E. Newman, "Community structure in social and biological networks," PNAS, vol. 99, no. 12, pp. 7821-7826, 2002.

[10] R. Guimera and L. A. N. Amaral, "Functional cartography of complex metabolic networks," Nature, vol. 433, no. 7028, pp. 895900, 2005.

[11] G. Palla, I. Derényi, I. Farkas, and T. Vicsek, "Uncovering the overlapping community structure of complex networks in nature and society," Nature, vol. 435, no. 7043, pp. 814-818, 2005

[12] G. Palla, A.-L. Barabási, and T. Vicsek, "Quantifying social group evolution," Nature, vol. 446, no. 7136, pp. 664-667, 2007.

[13] D. S. Bassett, N. F. Wymbs, M. A. Porter, P. J. Mucha, J. M. Carlson, and S. T. Grafton, "Dynamic reconfiguration of human brain networks during learning," Proceedings of the National Academy of Sciences, vol. 108, no. 18, pp. 7641-7646, 2011.

[14] M. Barigozzi, G. Fagiolo, and G. Mangioni, "Identifying the community structure of the international-trade multi-network," Physica A: Statistical Mechanics and its Applications, vol. 390, no. 11, pp. 2051 - 2066, 2011. [Online]. Available: http://www. sciencedirect.com/science/article/pii/S0378437111001129

[15] - Community Structure in the Multi-network of International Trade. Berlin, Heidelberg: Springer Berlin Heidelberg, 2011, pp. 163-175. [Online]. Available: http://dx.doi.org/10.1007/ 978-3-642-25501-4_17

[16] S. Fortunato, "Community detection in graphs," Physics Reports, vol. 486, no. 3-5, pp. $75-174,2010$.

[17] S. Fortunato and D. Hric, "Community detection in networks: A user guide," Physics Reports, vol. 659, pp. 1-44, 2016.

[18] A.-L. Barabási, N. Gulbahce, and J. Loscalzo, "Network medicine: a network-based approach to human disease," Nature reviews genetics, vol. 12, no. 1, p. 56, 2011.

[19] K.-I. Goh, M. E. Cusick, D. Valle, B. Childs, M. Vidal, and A.-L. Barabási, "The human disease network," Proceedings of the National Academy of Sciences, vol. 104, no. 21, pp. 8685-8690, 2007.

[20] A. Halu, M. De Domenico, A. Arenas, and A. Sharma, "The multiplex network of human diseases," bioRxiv, p. 100370, 2017.

[21] E. L. Huttlin, R. J. Bruckner, J. A. Paulo, J. R. Cannon, L. Ting, K. Baltier, G. Colby, F. Gebreab, M. P. Gygi, H. Parzen et al., "Architecture of the human interactome defines protein communities and disease networks," Nature, vol. 545, no. 7655, p. 505, 2017.

[22] M. D. Domenico, "Multilayer network modeling of integrated biological systems," Physics of Life Reviews, vol. In Press, 2018.

[23] H. Jeong, S. P. Mason, A.-L. Barabási, and Z. N. Oltvai, "Lethality and centrality in protein networks," Nature, vol. 411, no. 6833, p. $41,2001$.
[24] A. Vinayagam, T. E. Gibson, H.-J. Lee, B. Yilmazel, C. Roesel, Y. Hu, Y. Kwon, A. Sharma, Y.-Y. Liu, N. Perrimon et al., "Controllability analysis of the directed human protein interaction network identifies disease genes and drug targets," Proceedings of the National Academy of Sciences, vol. 113, no. 18, pp. 4976-4981, 2016.

[25] A.-L. Barabasi and Z. N. Oltvai, "Network biology: understanding the cell's functional organization," Nature reviews genetics, vol. 5, no. 2, p. 101, 2004.

[26] S.-H. Yook, Z. N. Oltvai, and A.-L. Barabási, "Functional and topological characterization of protein interaction networks," Proteomics, vol. 4, no. 4, pp. 928-942, 2004.

[27] J. Wu, T. Vallenius, K. Ovaska, J. Westermarck, T. P. Mäkelä, and S. Hautaniemi, "Integrated network analysis platform for proteinprotein interactions," Nature Methods, vol. 6, no. 1, p. 75, 2009.

[28] T. Gandhi, J. Zhong, S. Mathivanan, L. Karthick, K. Chandrika, S. S. Mohan, S. Sharma, S. Pinkert, S. Nagaraju, B. Periaswamy et al.," "Analysis of the human protein interactome and comparison with yeast, worm and fly interaction datasets," Nature genetics, vol. 38, no. 3, p. 285, 2006.

[29] T. Rolland, M. Tașan, B. Charloteaux, S. J. Pevzner, Q. Zhong, N. Sahni, S. Yi, I. Lemmens, C. Fontanillo, R. Mosca et al., "A proteome-scale map of the human interactome network," Cell, vol. 159, no. 5, pp. 1212-1226, 2014.

[30] M. E. J. Newman and M. Girvan, "Finding and evaluating community structure in networks," Phys. Rev. E, vol. 69, p. 026113, Feb 2004.

[31] M. E. J. Newman, "Modularity and community structure in networks," Proceedings of the National Academy of Sciences, vol. 103, no. 23, pp. 8577-8582, 2006.

[32] S. Fortunato and M. Barthelemy, "Resolution limit in community detection," Proceedings of the National Academy of Sciences, vol. 104, no. 1, pp. 36-41, 2007.

[33] B. H. Good, Y.-A. de Montjoye, and A. Clauset, "Performance of modularity maximization in practical contexts," vol. 81 , no. 4, p. 046106, Apr. 2010.

[34] J. Reichardt and S. Bornholdt, "Detecting fuzzy community structures in complex networks with a potts model," Physical Review Letters, vol. 93, no. 21, p. 218701, 2004.

[35] J. Duch and A. Arenas, "Community detection in complex networks using extremal optimization," Physical Review E, vol. 72, no. 2, p. 027104, 2005.

[36] L. Danon, A. Diaz-Guilera, J. Duch, and A. Arenas, "Comparing community structure identification," Journal of Statistical Mechanics: Theory and Experiment, vol. 2005, no. 09, p. P09008, 2005.

[37] E. A. Leicht and M. E. J. Newman, "Community structure in directed networks," Phys. Rev. Lett., vol. 100, p. 118703, Mar 2008.

[38] M. E. J. Newman, "Analysis of weighted networks," Phys. Rev. E, vol. 70, p. 056131, Nov 2004.

[39] M. J. Barber, "Modularity and community detection in bipartite networks," Phys. Rev. E, vol. 76, p. 066102, Dec 2007.

[40] V. Nicosia, G. Mangioni, V. Carchiolo, and M. Malgeri, "Extending the definition of modularity to directed graphs with overlapping communities," Journal of Statistical Mechanics: Theory and Experiment, vol. 2009, no. 03, p. P03024, 2009.

[41] M. Rosvall and C. T. Bergstrom, "Maps of random walks on complex networks reveal community structure," PNAS, vol. 105, no. 4, pp. 1118-1123, 2008.

[42] M. Rosvall, D. Axelsson, and C. T. Bergstrom, "The map equation," The European Physical Journal Special Topics, vol. 178, no. 1, pp. 1323, 2009.

[43] F. Battiston, V. Nicosia, and V. Latora, "Structural measures for multiplex networks," Phys. Rev. E, vol. 89, p. 032804, Mar 2014.

[44] M. De Domenico, A. Solé-Ribalta, E. Omodei, S. Gómez, and A. Arenas, "Ranking in interconnected multilayer networks reveals versatile nodes." Nature communications, vol. 6, p. 6868, 2015.

[45] E. Cozzo, M. Kivelä, M. De Domenico, A. Solé-Ribalta, A. Arenas, S. Gómez, M. A. Porter, and Y. Moreno, "Structure of triadic relations in multiplex networks," New Journal of Physics, vol. 17, no. 7, p. 073029, 2015.

[46] J. Iacovacci, C. Rahmede, A. Arenas, and G. Bianconi, "Functional multiplex pagerank," EPL (Europhysics Letters), vol. 116, no. 2, p. 28004, 2016.

[47] P. J. Mucha, T. Richardson, K. Macon, M. A. Porter, and J.-P. Onnela, "Community structure in time-dependent, multiscale, and multiplex networks," Science, vol. 328, no. 5980, pp. 876-878, 2010. 
[48] J.-C. Delvenne, S. N. Yaliraki, and M. Barahona, "Stability of graph communities across time scales," Proceedings of the National Academy of Sciences, vol. 107, no. 29, pp. 12 755-12 760, 2010.

[49] R. Lambiotte, J.-C. Delvenne, and M. Barahona, "Random walks, markov processes and the multiscale modular organization of complex networks," IEEE Transactions on Network Science and Engineering, vol. 1, no. 2, pp. 76-90, 2014.

[50] V. D. Blondel, J.-L. Guillaume, R. Lambiotte, and E. Lefebvre, "Fast unfolding of communities in large networks," Journal of Statistical Mechanics: Theory and Experiment, vol. 2008, no. 10, p. P10008, 2008.

[51] V. Carchiolo, A. Longheu, M. Malgeri, and G. Mangioni, Communities Unfolding in Multislice Networks. Berlin, Heidelberg: Springer Berlin Heidelberg, 2011, pp. 187-195. [Online]. Available: http://dx.doi.org/10.1007/978-3-642-25501-4_19

[52] M. De Domenico, A. Lancichinetti, A. Arenas, and M. Rosvall, "Identifying modular flows on multilayer networks reveals highly overlapping organization in interconnected systems," Phys. Rev. X, vol. 5, p. 011027, Mar 2015.

[53] M. De Domenico, A. Solé-Ribalta, S. Gómez, and A. Arenas, "Navigability of interconnected networks under random failures," Proceedings of the National Academy of Sciences, vol. 111, no. 23, pp. 8351-8356, 2014.

[54] L. Peel, D. B. Larremore, and A. Clauset, "The ground truth about metadata and community detection in networks," Science Advances, vol. 3, no. 5, 2017. [Online]. Available: http://advances.sciencemag.org/content/3/5/e1602548

[55] E. Ziv, M. Middendort, and C. H. Wiggins, "Information-theoretic approach to network modularity," Phys. Rev. E, vol. 71, p. 046117, Apr 2005.

[56] M. Rosvall and C. T. Bergstrom, "An information-theoretic framework for resolving community structure in complex networks," Proceedings of the National Academy of Sciences, vol. 104, no. 18, pp. 7327-7331, 2007.

[57] T. P. Peixoto, "Parsimonious module inference in large networks," Phys. Rev. Lett., vol. 110, p. 148701, Apr 2013.

[58] , "Entropy of stochastic blockmodel ensembles," Physical Review E, vol. 85, no. 5, May 2012. [Online]. Available: http://opus.bath.ac.uk/52703/

[59] C. Aicher, A. Z. Jacobs, and A. Clauset, "Learning latent block structure in weighted networks," Journal of Complex Networks, vol. 3, no. 2, pp. 221-248, 2015.

[60] M. De Domenico, M. A. Porter, and A. Arenas, "Muxviz: a tool for multilayer analysis and visualization of networks," Journal of Complex Networks, vol. 3, no. 2, p. 159, 2015.

[61] C. Stark, B.-J. Breitkreutz, T. Reguly, L. Boucher, A. Breitkreutz, and M. Tyers, "Biogrid: a general repository for interaction datasets," Nucleic Acids Research, vol. 34, no. suppl 1, pp. D535-D539, 2006.

[62] A. Subramanian, P. Tamayo, V. K. Mootha, S. Mukherjee, B. L. Ebert, M. A. Gillette, A. Paulovich, S. L. Pomeroy, T. R. Golub, E. S. Lander, and J. P. Mesirov, "Gene set enrichment analysis: A knowledge-based approach for interpreting genome-wide expression profiles," Proceedings of the National Academy of Sciences, vol. 102, no. 43, pp. 15545-15550, 2005.

[63] A. Liberzon, A. Subramanian, R. Pinchback, H. Thorvaldsdttir, P. Tamayo, and J. P. Mesirov, "Molecular signatures database (MSigDB) 3.0," Bioinformatics, vol. 27, no. 12, pp. 1739-1740, 2011.

[64] A. Liberzon, C. Birger, H. Thorvaldsdttir, M. Ghandi, J. P. Mesirov, and P. Tamayo, "The Molecular Signatures Database Hallmark Gene Set Collection," Cell Systems, vol. 1, no. 6, pp. 417-425, 2015.

[65] P. Jaccard, "Lois de distribution florale dans la zone alpine," Bulletin de la Société Vaudoise des Sciences Naturelles, vol. 38, pp. 69-130, 1902.

[66] _ "Nouvelles Recherches Sur La Distribution Florale," Bulletin de la Société Vaudoise des Sciences Naturelles, vol. 44, pp. 223-270, 1908. 